Acta Crystallographica Section B

Structural

Science

ISSN 0108-7681

\section{Richard E. Marsh}

The Beckman Institute, California Institute of Technology, Pasadena, CA 91125, USA

Correspondence e-mail: rmarsh@caltech.edu

\section{Space group Cc: an update}

A recent survey of the Cambridge Structural

Received 6 January 2004

Accepted 18 February 2004 Database, CSD [Allen (2002). Acta Cryst. B58, 380388 ], shows that the percentage of incorrect assignments of the space group $C c$ has remained at about $10 \%$ since the last survey in 1997.

\section{Introduction}

The monoclinic space group No. 9 ( $C c$, or other settings of it) seems to be a particularly bothersome one. Two previous surveys (Baur \& Kassner, 1992; Marsh, 1997) have shown that approximately $10 \%$ of the structures reported in space group No. 9 and included in the Cambridge Structural Database (Allen, 2002; CSD) are properly described in space groups of higher symmetry $(C 2 / c$, usually, but occasionally in higher Laue groups). Since those two surveys, there have been major advances in structure-solving programs and especially in checking routines which can easily search for higher symmetry once a structure has been solved. Nevertheless, a new survey of the CSD indicates that errors in the assignment of space group No. 9 remain at approximately the $10 \%$ level.

\section{Experimental}

In June 2003 A. L. Spek (Private Communication) surveyed the CSD (October, 2002 release plus two supplementary updates in 2003), using the program PLATON-MISSYM (Spek, 2003); this survey turned up over 5000 entries for which the documented space group seemed questionable. A subsequent survey based only on entries for space group No. 9 ( $C c$ or its equivalent in other settings) and which included a third 2003 update of the CSD turned up 534 questionable structures of a total of 3111 entries. (Many of these 3111 entries were either duplicates or contained little structural information; the number of independent, presumably valid entries is probably around 2800.) Of the 534 questionable $C c$ structures, 170 have already been revised. (The revised structures are included as separate entries in the CSD, with different extensions to the Refcode.) I have now examined more closely the remaining questionable entries and identified 164 additional structures whose space groups should be revised to ones of higher symmetry. ${ }^{1}$ These 164 structures are listed in a supplementary table, and the revised coordinates have been submitted to the CSD. ${ }^{2}$

\section{Comments}

In the large majority of cases (134) the revised space group is $C 2 / c$, entailing the addition of a center of inversion. As is usual in such situations, the non-centrosymmetric descriptions in space group $C c$ tend to be severely distorted, with bond lengths differing from expected values by many e.s.d.'s; it is not unusual to find aromatic $\mathrm{C}-\mathrm{C}$ distances as short as $1.2 \AA$ or as long as $1.6 \AA$. (In one instance - Refcode XAJWAG - unusual C-C distances apparently convinced a hydrogen-positioning routine that two aromatic $\mathrm{C}$ atoms were aliphatic in nature and hence that they should be doubly protonated.) The revision of the structure of CIFHII from $C c$ to $C 2 / c$ strongly suggests that a single ' $\mathrm{HCl}$ ' molecule should be replaced by two

\footnotetext{
${ }^{1}$ The original publication was always consulted. In several instances, not included among the 164 , the authors recognized the ambiguity in space group and had valid reason for selecting $C c$.

${ }^{2}$ Supplementary data for this paper are available from the IUCr electronic archives (Reference: BK0142). Services for accessing these data are described at the back of the journal.
} 
water molecules related by the added $C_{2}$ axis. In the case of PMPDCU I have rerefined the structure in the space group $C 2 / c$, based on the 1117 intensities used in the original investigation (Pajunen \& Pajunen, 1979); $R$ was unchanged, at 0.051 , despite an approximate halving of the number of parameters. In the revised structure the coordinated $\mathrm{NO}_{2}$ group lies on a $C_{2}$ axis and is disordered, being $\mathrm{N}$-bonded to the $\mathrm{Cu}$ atom approximately $65 \%$ of the time and bonded (symmetrically) by the two $\mathrm{O}$ atoms $35 \%$ of the time. The uncoordinated $\mathrm{NO}_{2}^{-}$ counterion is also disordered.

After $C 2 / c$, the most common spacegroup revision is to $F d d 2$, in 23 cases; Ima 2 is represented twice and $A m a 2, A b a 2, P 3 c$, $R 3 c$ and $P \overline{3} 1 c$ once each. Only in the case of $P \overline{3} 1 c$ (ROMTIW) does the Laue-group revision carry significant changes in molecular geometry; here, the matching of coordinates across the added center of symmetry leads to r.m.s. deviations of $c a 0.05 \AA$, while subsequent matching around the threefold axis shows deviations of only $c a 0.01 \AA$, as expected (Schomaker \& Marsh, 1979).

In all but a few cases (probably less than a dozen) the 164 corrections noted here represent structures that appeared in the CSD subsequent to the prior survey of this space group (Marsh, 1997); of 280 Cc entries in the three latest (2003) updates of the CSD, 26 are revised either here or in other entries. Thus, it appears that the rate of error in this space group, which was originally estimated at 'possibly over $10 \%$ ' by Baur \& Kassner (1992) and confirmed by Marsh (1997), seems to be remaining relatively constant. This is discouraging news, particularly in view of the many advances in data collection, structure refinement and symmetry-checking routines. (There appear to be no incorrect space-group assignments of small-molecule structures in recent issues of Acta Crystallographica, which routinely checks for missed symmetry.) It is quite possible that the improvements in structuresolving automation have been counterbalanced by an increasing reluctance of individual experimentalists and scientific journals to assess the reliability of crystallographic results.

I greatly appreciate the help and advice of L. Henling and A. L. Spek in many aspects of this work.

\section{References}

Allen, F. H. (2002). Acta Cryst. B58, 380-388. Baur, W. H. \& Kassner, D. (1992). Acta Cryst. B48, 356-369.

Marsh, R. E. (1997). Acta Cryst. B53, 317-322.

Pajunen, A. \& Pajunen, S. (1979). Acta Cryst. B35, 3058-3060.

Schomaker, V. \& Marsh, R. E. (1979). Acta Cryst. B35, 1933-1934.

Spek, A. L. (2003). J. Appl. Cryst. 36, 7-13. 